\title{
Percepción de la comunidad universitaria sobre la calidad de vida en Venezuela: el caso Universidad Metropolitana
}

\author{
Gloria Marina Lopez ${ }^{1}$, Yuherqui Ayari Guaimaro², Magaly Rafaela Couret ${ }^{3}$ \\ Universidad Metropolitana - Caracas
}

\begin{abstract}
Artículo científico
Material original autorizado para su primera publicación en el Journal de Ciencias Sociales, Revista Académica de la Facultad de Ciencias Sociales de la Universidad de Palermo.
\end{abstract}

\section{Recibido 15-8-2017}

Aceptado 10-9-2017

\section{Resumen}

La calidad de vida es un concepto experiencial, dinámico, flexible y significativo, que refleja el punto de vista individual del bienestar y funcionamiento de cada sujeto (Kreitler y Nive, 2007; Tonon, 2003). Es definido por los autores de distintas maneras, ya que depende del contexto y de las personas, es multidimensional y no se basa únicamente en una medición global sino en dominios específicos identificados como constitutivos de ella. Las condiciones de vida están no solo en función de la persona, sino también de los factores contextuales que condicionan de cierto modo la calidad de vida. En la comunidad universitaria, en el caso de la Universidad Metropolitana, se han manifestado cambios en el sentir, hacer y convivir dentro de ella. De ahí surge el valorar de la percepción de la comunidad unimetana (profesores, estudiantes y personal administrativo) sobre su calidad de vida enmarcada en el contexto actual de la situación del país. Sobre la base de las investigaciones de Schalock (1997) y Tonoin (2003), se utilizó un enfoque cualitativo de investigación de campo tipo exploratoria y la recolección de datos se realizó mediante la adaptación de la escala Gencat, que consta de 69 ítems, distribuidos en ocho sub-escalas, denominadas dimensiones de la Calidad de Vida. El análisis de los datos reflejó que las dimensiones de relaciones interpersonales, desarrollo personal, autodeterminación e inclusión social son las mejor valoradas por todos los grupos, en contraste con otras,

\footnotetext{
${ }^{1}$ Profesora e investigadora. Directora del Centro de Investigaciones para la Infancia y la Familia (CENDIF) - Facultad de Ciencias y Artes,Universidad Metropolitana, Caracas, Venezuela. Correo electrónico:glopez@unimet.edu.ve

${ }^{2}$ Profesora e investigadora del Centro de Investigaciones para la Infancia y la Familia (CENDIF) Facultad de Ciencias y Artes. Universidad Metropolitana. Carcas, Venezuela.

${ }^{3}$ Profesora e investigadora del Centro de Investigaciones para la Infancia y la Familia (CENDIF) Facultad de Ciencias y Artes. Universidad Metropolitana. Caracas. Venezuela.
} 
valoradas en un nivel muy bajo, como las de derechos en los estudiantes; bienestar emocional y material en el personal administrativo, y bienestar físico en los profesores. La percepción de la calidad de vida en los tres grupos fue de baja a media. Se sugiere el diseño y desarrollo de alternativas que mejoren la percepción y las condiciones sociales en las que se encuentra la comunidad universitaria tomando en cuenta las condiciones del país.

Palabras clave: Calidad de vida; Percepción; Grado de bienestar.

\section{Abstract}

Quality of life is an experiential, dynamic, flexible and meaningful concept that reflects the individual's view of the well-being and functioning of each subject (Kreitler and Nive, 2007; Tonon, 2003). It is defined by the authors in different ways, since it depends on the context and the people, it is multidimensional and is not based only on a global measurement but also on specific domains identified as constitutive of it. The living conditions are not only defined in terms of the person, but also of the contextual factors that condition the quality of life in a certain way. In the university community, in the case of the Metropolitan University, there have been changes in the feeling, doing and coexisting within it. From therearises the evaluation of the perception of the unimetan community (teachers, students and administrative staff) on its quality of life in the current context of the country's situation. Based on Schalock's(1997) and Tonon's (2003)Research, a qualitative field research approach of exploratory type was used and the data collection was performed by adapting the Gencat scale, consisting of 69 items, distributed in eight sub-scales, called dimensions of the Quality of Life. The analysis of the data showed that the dimensions of interpersonal relationships, personal development, self-determination and social inclusion are the best valued by all groups, in contrast to others, valued at a very low level, such as rights in students; emotional well-being and welfare in the administrative staff, and physical well-being in teachers. The perception of the quality of life in the three groups was low to medium. It is suggested the design and development of alternatives that improve the perception and social conditions in which the university community is found, taking into account the conditions of the country.

Key words: Quality of life; Perception; Well-being Degree.

\section{Introducción}


Cuando nos referimos al término calidad de vida pareciera que no existe un consenso sobre su definición, medición y resultados. Se evidencian diferentes concepciones que varían desde una perspectiva cuantitativa medida por los bienes y servicios existentes y por la satisfacción de las necesidades de los seres humanos; una cualitativa medida desde la subjetividad sustentada en el sentir de la persona, y otra de carácter multidimensional, percibida como la garantía de las condiciones en las que viven los miembros de una comunidad o sociedad.

Schalock y Verdugo (2007) señalan, a través de su experiencia de revisión bibliográfica internacional, que las dimensiones del concepto calidad de vida se refieren a las relaciones interpersonales, inclusión social, desarrollo personal, bienestar físico, autodeterminación, bienestar material, bienestar emocional y derechos.

En la Universidad Metropolitana (Unimet), a través de los años, se han manifestado cambios en el sentir, hacer y convivir dentro de ella, situación que depende no solo de cada persona sino también del contexto. En este sentido, nos interesa conocer desde una perspectiva integral la percepción de la calidad de vida de los unimetanos y reflejarlas circunstancias que permitirían obtener buenas condiciones de vida con un alto grado de bienestar.

\section{Antecedentes}

Es importante reconocer la multidimensionalidad de la Calidad de Vida. La reflexión sobre bienestar y felicidad se remonta a Platón y Aristóteles. Existe gran dispersión y diversidad sobre el concepto de Calidad de Vida (CdV), debido a que es un término multidisciplinario (Haas, 1999). El concepto de CdVes estudiado por tres ramas de las ciencias: Economía, Medicina y Ciencias Sociales. Cada una promueve un punto de vista diferente respecto a cómo debiera ser conceptualizada (Cummins, 2005).

Durante los años cincuenta y comienzo de los sesenta, se desarrolló un creciente interés por conocer el bienestar humano y se incrementó la preocupación por las consecuencias de la industrialización de la sociedad, de allí surgió la necesidad de medir esta realidad a través de datos objetivos. Es así como desde las Ciencias Sociales se inició el desarrollo de los indicadores sociales, con estadísticos que permitieron medir datos y hechos vinculados al bienestar social de una población. Estos indicadores tuvieron su propia evolución; en un primer momento fueron una referencia de las condiciones objetivas, de tipo económico y social, y, en un segundo momento, llegaron a contemplar elementos subjetivos (Arostegui, 1998).

A lo largo de los años la calidad de vida se ha asociado al desarrollo económico y a la adquisición de bienes materiales. Según Blanco (1985) el error ha estado en vincular la 
calidad de vida al puro desarrollo económico, ya que se habían olvidado los aspectos más cualitativos del concepto.

El concepto evolucionó hacia el año 1975 y alcanzó su máximo desarrollo a lo largo de los años ochenta. Se originó en el campo de la Medicina y más tarde se extendió al de la Sociología y la Psicología. El autor García-Riaño (1991) alega que no existen referencias históricas bibliográficas del concepto calidad de vida en sí, y es por ello por lo que para hacer un poco de historia tenemos que recurrir a sus términos afines, tales como salud, bienestar y felicidad.

Como podemos observar, la concepción de calidad de vida, a lo largo de su desarrollo histórico, se ha caracterizado por su continua ampliación. Inicialmente, solo hacía referencia al cuidado de la salud personal; luego se convirtió en la preocupación por la salud personal e higiene pública, y más tarde se incluyeron los derechos humanos, con énfasis en los derechos laborales y ciudadanos. Más tarde, se agregó la capacidad de acceso a los bienes económicos y se extendió a la preocupación por la experiencia del sujeto en su vida social y su actividad cotidiana.

En los países desarrollados el estudio de calidad de vida tuvo un auge bastante considerable a partir de la segunda mitad de los años sesenta. Esto se ha materializado en múltiples estudios globales que han medido la calidad de vida mediantediversos indicadores sociales con énfasis en el bienestar percibido, medido a través del juicio de satisfacción y felicidad (Campbell, Converse y Rodgers, 1976; Andrews y Withey, 1976). La naturaleza compleja del concepto calidad de vida dificulta su definición, de allí que algunos autores, como Calman (1987) queanalizó más de doscientos trabajos sobre el tema, afirma que es un concepto global, inclusivo y multidisciplinar.

Autores como Levi y Anderson (1980) proponen asumir los nueve componentes (salud, alimentación, educación, trabajo, vivienda, seguridad social, vestido, ocio y derechos humanos) propuestos por las Naciones Unidas, por considerar que el conjunto de los mismos constituye el nivel de vida de los pueblos y las personas en general. Andrews y Whithey (1976) definen la Calidad de Vida como el reflejo subjetivo de la evaluación que cada individuo hace de la condición sí mismo, y destacan que no es el reflejo de condiciones observables, reales y objetivas.

Por su parte, Lawton (1975) la define como el conjunto de evaluaciones que el individuo hace sobre cada uno de los dominios de su vida actual. Para Shin y Johnson (1978) es la posesión de los recursos necesarios para la satisfacción de las necesidades y deseos individuales, la participación en aquellas actividades que favorecen el desarrollo personal y la comparación del estado de satisfacción con los otros. Campbell (1976) agrega que los indicadores objetivos socioeconómicos no bastan para generar el estado de satisfacción, sino que además es necesario desearlos, por lo que no es extraño que surjan 
discrepancias cuando se trata de determinar quién debe evaluar o definir lo que es la calidad de vida.

La Organización Mundial de la Salud (OMS) (1996) ha definido la calidad de vida como la percepción que cada individuo tiene de su posición en la vida en el contexto del sistema cultural y de valores en el que vive, en su relación con sus metas, expectativas, estándares y preocupaciones. Osorio, Torrejón y Anigstein (2011) hacen notar que esta definición se centra en aspectos subjetivos de la calidad de vida. Por su parte Urzúa y Caqueo-Urizar (2012) agregan que la calidad de vida se puede definir como el nivel percibido de bienestar derivado de la evaluación que realiza un individuo de elementos objetivos y subjetivos en distintas dimensiones de su vida, al igual que Nava (2012).

Según Tonon (2009), para el año 1998 se planteó la necesidad de separar los componentes objetivos y subjetivos de la calidad de vida, por la consideración de que el bienestar personal es un concepto más restringido que el de calidad de vida y es un vehículo para entender a los sujetos, sus sentimientos, motivaciones, pensamientos y acciones. Sin embargo, el concepto de bienestar, que significa tener buenas condiciones de vida objetivas y un alto grado de bienestar subjetivo, es usado como sinónimo de calidad de vida y representa un término multidimensional de las políticas sociales (Palomba, 2002). A la parte subjetiva de la calidad de vida se le ha dado énfasis, no solo para diferenciar el grado de desarrollo entre países, sino también por su forma de medición. En este sentido, se ha desarrollado una iniciativa en algunos países de América Latina, —entre ellos Cuba, Venezuela y Ecuador-, que parte de la idea que el modelo de desarrollo es distinto a los modelos neoliberales (Rojas y Martínez, 2012).

Esto nos demuestra que, a lo largo de su desarrollo histórico, el concepto de calidad de vida se ha caracterizado por su continua ampliación, que va desde el cuidado de la salud personal y aspectos de la salud pública, así como los derechos humanos, laborales y ciudadanos, hasta la capacidad de acceso a bienes económicos y aspectos de la vida social. El término acaba por ser profundamente complejo, pero contradictoriamente muy frágil, lo que posibilita una gran diversidad de interpretaciones de los diferentes autores. Esto nos lleva a concluir que ambos aspectos, objetivos y subjetivos, son procesos intrínsecamente interrelacionados.

\section{Bases teóricas}

Para el presente estudio es necesario comprender que el concepto calidad de vida se basa no solo en los ámbitos materiales sino también en ámbitos más transcendentales, dados por las condiciones necesarias para el desarrollo de las capacidades. Este concepto también es utilizado para referirse al crecimiento y desempeño de la persona dentro de la 
sociedad, a las buenas oportunidades que pueda tener en distintos aspectos y, sobre todo, a la satisfacción de vencer las condicionantes que se presentan en la vida.

Investigadores a nivel mundial señalan que en las últimas décadas la calidad de vida ha sido más y más el foco de la investigación en discapacidad y campos relacionados. El significado de "calidad está asociado con las características humanas y los valores positivos como la felicidad, el éxito, la salud, la riqueza y la satisfacción. Por su parte, "de vida" indica que el concepto concierne a la misma esencia o a aspectos esenciales de la vida humana. Este significado explica por qué el concepto de calidad de vida está impactando en el campo de la discapacidad, ya que promueve un nuevo enfoque.

Según Robert Schalock (1997) - un excelente investigador de la Discapacidad Intelectual - la calidad de vida se define como un concepto que refleja las condiciones de vida deseadas por una persona en relación con ocho necesidades fundamentales que representan el núcleo de las dimensiones de la vida de cada uno. Estas dimensiones son: bienestar emocional, relaciones interpersonales, bienestar material, desarrollo personal, bienestar físico, autodeterminación, inclusión social y derechos. En este modelo todas y cada una de ellas tienen la posibilidad de ser mejoradas en cualquier persona, independientemente de que las acompañe o no la condición de retraso mental con una necesidad de apoyo más o menos generalizado.

Por otra parte, Shalock (2005) nos aporta los principios de la conceptualización, medida y aplicación de la Calidad de Vida. Sobre la conceptualización, agrega que es multidimensional, que está influida por la interacción de factores personales y ambientales, que tiene componentes objetivos y subjetivos, y que se mejora con la autodeterminación, los recursos, el propósito de vida y un sentido de pertenencia. Respecto a la medida, considera el contexto de los ambientes físico, social y cultural, e incluye medidas de experiencias tanto comunes como únicas para los seres humanos. En cuanto a la aplicación, debe estar sostenida por evidencias y sus principios deben ser la base de intervenciones y apoyos.

En esta evolución del concepto calidad de vida, progresivamente se ha generado un acuerdo de definición multidimensional.Se ha señalado que, además, debe ser visto desde una perspectiva jerárquica, en la que se debe tener en cuenta tres factores: la calidad de vida es subjetiva, las dimensiones centrales son valoradas por las personas en forma diferente y el valor adscrito a cada una varía a lo lago de la vida de estas personas.

Topolski, Edwards y Patrick (2002) señalan que el efecto de las relaciones sociales y con el medio se encuentra mediatizado por la particular mirada de cada sujeto, y es por eso que la manera en que cada uno interpreta su contexto y situación se convierte en la llave que determina su calidad de vida. Asimismo, los efectos que produce el cambio en las circunstancias de vida de una persona son percibidos de forma diferente por quien ha vivido 
la experiencia que por quienes han sido observadores pasivos (Gullone y Cummins, 2002). Dado que las personas evalúan de manera diferente las circunstancias de su vida, en relación a sus expectativas, valores y experiencias previas, los estudios sobre la calidad de vida reconocen la importancia de esas diferencias (Bramston, 2002).

Cabe destacar la amplitud que ha tomado la definición de la Calidadde Vida, ya que en 1995 se fundóla International Society for Quality of Life Studies (ISQOLS) con el objetivo general de incentivar el desarrollo de estudios sobre este tema a nivel mundial, y de estimular investigaciones interdisciplinarias en los campos de la política, lo social, el comportamiento, la medicina y otras disciplinas dedicadas al desarrollo y el medio ambiente (Tonon, 2003).

Casas (1999) define la Calidad de Vida como "las percepciones, aspiraciones, necesidades, satisfacciones y representaciones sociales que los miembros de todo conjunto social experimentan en relación con su entorno y la dinámica social en que se encuentran inmersos, incluyendo los servicios que se les ofrecen y las intervenciones sociales de las que son destinatarios y que emanan de las políticas sociales".

La calidad de vida está entonces condicionada por la estructura social, que puede ser considerada en términos de características demográficas, pautas culturales, características psicosociales de la comunidad, así como por las características de las instituciones, tanto del ámbito estatal como del privado, que desarrollan su accionar en ese contexto (Ferris, 2006). En este sentido Cummins y Cahill (2000) consideraron que la calidad de vida se define culturalmente .

Para Tonon (2003), que coincide con Kreitler y Nive (2007), la calidad de vida es un concepto subjetivo, que refleja el punto de vista individual del bienestar y funcionamiento de cada sujeto. Es un constructo fenomenológico que provee una imagen de la situación sin explicar por qué ni cómo surgió.Es experiencial y evaluativo, ya que presenta un juicio sin ningún intento de relacionarlo con variables objetivas.Es dinámico, flexible y significativo para cada sujeto, y es multidimensional, ya que no se basa solo en una medición global, sino en dominios específicos identificados como constitutivos de la calidad de vida.

\section{Contexto venezolano}

Hacia el año 2015, según estudios realizados desde Provea (Programa Venezolano de Educación-Acción en Derechos Humanos), había al menos 23 millones de venezolanos con dificultades para satisfacer sus necesidades a partir del ingreso. Esto equivale a que el $75 \%$ de la población se encuentra en situación de pobreza, más que en los registros de 1990 y 1999. El aumento general de los precios, que durante 2015 alcanzó 180\%, en el sector de los alimentos, llegó al 315\%, según cifras del Banco Central de Venezuela. Los venezolanos han dejado de consumir las tres comidas diarias y han llegado a modificar su 
dieta. Aumentaron la ingesta de carbohidratos y la tasa de desnutrición se incrementó un $13,4 \%$ y de la de sobrepeso, 6,8\% con respecto al año 2014. En cuanto al acceso a la salud, la organización Provea (2015) reporta que recibió 3719 denuncias (40\% más que el año anterior), específicamente sobre la falta de medicamentos y equipos médicos. Destacan que durante el año 2015 se mantuvo la impunidad estructural y la exclusión por razones políticas.

En el año 2016, las universidades UCAB, UCV y USB efectuaron la Encuesta Condiciones de Vida de la Población Venezolana (ENCOVI). Su finalidad fue contribuir al conocimiento de las condiciones de vida de la población en la actualidad y a la orientación del debate sobre las estrategias gubernamentales en política social en un contexto de recesión económica y de persistente conflictividad política. Ella informa que la inseguridad y la violencia son problemáticas cuya creciente intensidad se ha traducido en el menoscabo de la calidad de vida de la población venezolana, en la medida en que se ha coartado su derecho a la vida, a sus bienes y a la propiedad. Agrega que las personas perciben que en el último tiempo se ha incrementado la violencia tanto en el país como en su comunidad y la población en general reporta haber sido víctima de algún delito. Existe un elevado temor de ser atacado o robado, el $76 \%$ de las muertes violentas son de personas con menos de 35 años. Por ello la población ha modificado sus actividades en la calle y ha disminuido la confianza en los cuerpos policiales, en la justicia penal y en la gestión del gobierno en materia de seguridad.

Por otro lado, la ENCOVI de 2016 reporta que entre 2014 y 2016 el porcentaje de hogares en condición de pobreza pasó del 48,4\% al 81,8\% y los hogares en pobreza extrema pasaron del $23,6 \%$ al 51,5\%. Existe inseguridad alimentaria en el 93,3\% de los hogares venezolanos. En materia educativa, el 24,93\% de la población de entre 3 y 24 años no asiste a un centro de enseñanza y con respecto al área de salud, más del $63 \%$ de la población no tiene seguro de atención médica.

Objetivo general: valorar la percepción que tiene la comunidad unimetana (profesores, estudiantes y personal administrativo) sobre su calidad de vida enmarcada en el contexto actual de la situación del país.

\section{Objetivos específicos:}

- Describir la percepción que tienen los diferentes grupos de la comunidad unimetana sobre su calidad de vida.

- Comparar, entre los grupos estudiados, la percepción que tienen sobre su calidad de vida.

- Proponer acciones que incidan favorablemente en la calidad de vida de la comunidad unimetana. 
Metodología: enfoque cualitativo de investigación de campo tipo exploratoria, con una estrategia que aborda la naturaleza subjetiva de la calidad de vida.

Instrumento de recolección de datos: Se utilizó la escala GENCAT de calidad de vida elaborada por Verdugo, Arias, Gómez y Schalock, R. L (2009). Se trata de un cuestionario autoadministrado, donde el encuestado responde a cuestiones observables sobre la calidad de vida, que consta de 69 ítems enunciados en tercera persona, con formato declarativo y organizados en ocho dimensiones (Schalock y Verdugo, 2003): bienestar emocional (BE), bienestar físico (BF), bienestar material (BM), desarrollo personal (DP), autodeterminación $(A U)$, relaciones interpersonales (RI), inclusión social (IS) y derechos (DE).

El cuestionario proporciona un índice global de calidad de vida, así como puntuaciones en cada una de las ocho dimensiones y un perfil de la calidad de vida de la persona. Se responde utilizando una escala de frecuencia de cuatro opciones ('nunca o casi nunca', 'a veces', 'frecuentemente' y 'siempre o casi siempre').

Diseñada para validar la calidad de vida en personas con discapacidad, $\mathrm{VIH}$, adultos mayores y personas en general, incluye puntuaciones directas, puntuación estándar de cada dimensión, índice de calidad de vida y percentil del índice de calidad de vida.

\section{Bases empíricas:}

1. Se han realizado diferentes estudios para evaluar las propiedades psicométricas del instrumento. Algunos de los resultados obtenidos indican un buen nivel de confiabilidad, con valores de alfa de Cronbach .749 .853. Según el modelo de Escalas de Clasificación la confiabilidad es alta, con valores superiores a .95 en todas las dimensiones. En todo caso, la limitación más importante del estudio fue el tamaño de la muestra, lo que disminuye su confiabilidad (Benito, Mora y Macía, 2016).

2. Otros estudios han arrojado valores entre .915 y .931, así lo confirman Henao, Verdugo y Córdoba (2015) en una versión de la escala INICO-FEAPS adaptada a la población colombiana con discapacidad. Dicha escala presentó adecuados niveles de validez y fiabilidad.

3. En Venezuela, el Instituto Nacional de Capacitación Educativa Socialista (INCES), ha enfocado el Programa Nacional de Aprendizaje (PNA) a mejorar la calidad de vida de los estudiantes que participan en este programa social. El objetivo fue diseñar un modelo de estimación de la calidad de vida de los egresados del programa nacional de aprendizaje del INCES para el período 1985-2014 en el estado de Cojedes (Venezuela). Se trata de una investigación de campo de carácter descriptivo, con diseño no experimental y una muestra constituida por 278 egresados. Se considera trabajar con una muestra por estratos para cada 10 años, con selección sistemática por cada estrato dondea cada estrato le 
corresponden 91, 66 y 121 egresados, respectivamente. Para evaluar el Índice de la Calidad de Vida, se tomó como base el Modelo de Contreras y Cordero (1994), y el recientemente modificado por Jaimes y Carballo (2014). Es pertinente acotar que esta modificación tuvo como referente el Manual de Aplicación de la Escala GENCAT de Calidad de Vida, diseñado por Verdugo et al. (2009). El análisis estadístico fue univariado y multivariado, lo cual permitió analizar simultáneamente un conjunto de datos de diversas variables medidas para cada individuo, a través de la regresión logística ordinal que permitió generar el modelo que mejor explica la calidad de vida de los egresados (Raymundo y Jaimes, 2015).

Validez del instrumento seleccionado: los ítems se refirieron a cuestiones observables, concretas y fáciles de comprender. La corrección se realizó basada en el baremo del apartado A, correspondiente a la muestra general (válida para cualquier persona) con las siguientes dimensiones: bienestar emocional (BE), relaciones interpersonales (RI), bienestar material (BM), desarrollo personal (DP), bienestar físico (BF), inclusión social (IS), autodeterminación (AU) y derechos (DE).

Población: la comunidad que constituye la población de la Universidad Metropolitana, profesores con dedicación a tiempo completo, profesores con dedicación convencional o por horas, personal administrativo y estudiantes. Total de población encuestada: 231, distribuidos en una muestra representativa de cada grupo que integra la comunidad unimetana, como se indica a continuación: estudiantes (177), personal administrativo (30), profesores a tiempo convencional (14),[4] profesores a tiempo completo (10).

Consideraciones éticas: la presente investigación respetó la confidencialidad de los datos de los individuos encuestados, tanto en las fases de recolección de datos como al momento de analizar los resultados. Por lo que respecta a la participación de todos los agentes en la investigación, se ha tenido especial cuidado de ofrecer información exhaustiva, clara, veraz y precisa sobre la misma, de obtener el consentimiento voluntario de los colaboradores, yde mantener el trato respetuoso. Por otra parte, se efectuaron los trámites pertinentes para la solicitud de permisos y autorizaciones ante las instancias necesarias. Se espera que la institución considere los datos que le puedan ser útiles para establecer mejoras en la calidad de vida de los involucrados.

Resultados: a continuación, se presentan los datos recolectados en cada uno de los diferentes grupos que componen la muestra.

\section{Estudiantes}


Tabla 1. Distribución de estudiantes por sexo y grupo de edad

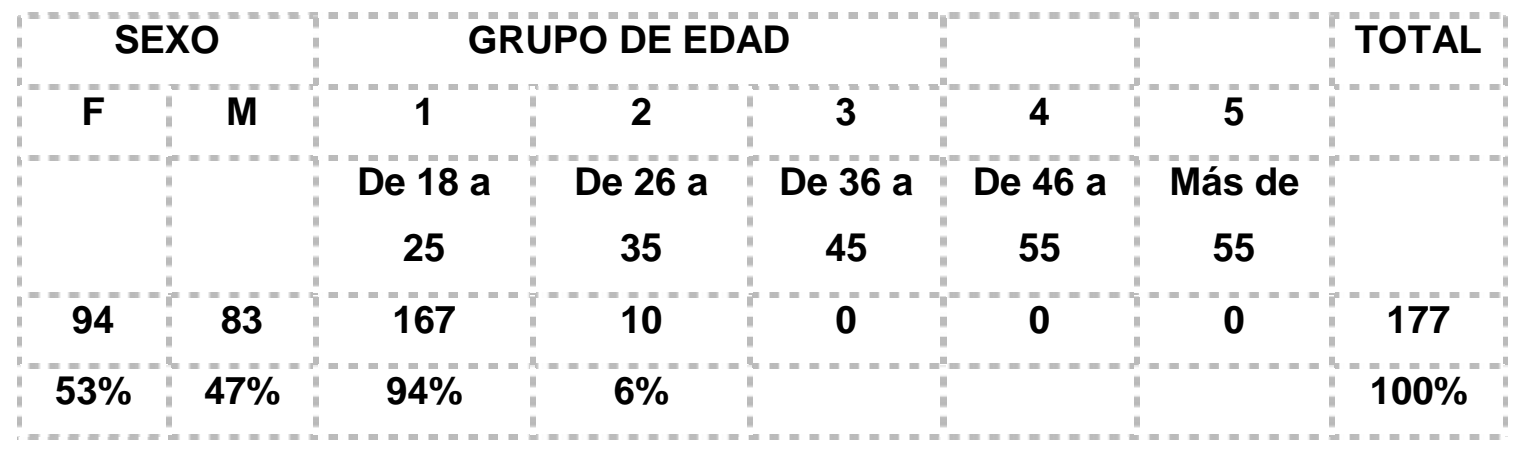

El total de estudiantes encuestados fue de 177; 94 del sexo femenino, correspondiente al $53 \%$ y 83 del sexo masculino, para un $47 \%$. Se distribuyeron entre los grupos de edad de18 a 25 y de 26 a 35, 167 (94\%) y $10(6 \%)$ respectivamente.

Tabla 2. Puntuación Directa Total/Puntuación Estándar/Percentil de las Dimensiones/Estudiantes

\begin{tabular}{|c|c|c|c|}
\hline Dimensiones & Puntuaciones & Puntuaciones & $\begin{array}{l}\text { Percentiles } \\
\text { de }\end{array}$ \\
\hline de Calidad de & directas & & las \\
\hline Vida & totales & estándar & dimensiones \\
\hline Bienestar & & & \\
\hline emocional & 22 & 9 & 37 \\
\hline Relaciones & & & \\
\hline interpersonales & 34 & 14 & 91 \\
\hline Bienestar material & 28 & 9 & 37 \\
\hline Desarrollo & & & \\
\hline Personal & 28 & 14 & 91 \\
\hline Bienestar físico & 26 & 8 & 25 \\
\hline Autodeterminación & 34 & 14 & 91 \\
\hline Inclusión social & 26 & 12 & 75 \\
\hline Derechos & 28 & 4 & 2 \\
\hline \multicolumn{2}{|c|}{ Puntuación estándar total } & 84 & \\
\hline \multicolumn{2}{|c|}{ Îndice de Calidad de Vida (PEC) } & 104 & \\
\hline \multicolumn{2}{|c|}{ Percentil del índice de Calidad de } & & \\
\hline & Vida & 60 & \\
\hline
\end{tabular}


En la tabla 2 podemos observar que los estudiantes alcanzaron una puntación estándar total de 84 , obtuvieron un índice de calidad de vida de 104 y un percentil del índice de calidad de vida de 60 .

\section{Gráfico 1. Representación gráfica de la Puntuación Directa Total/Puntuación} Estándar/Percentil de las Dimensiones/Estudiantes

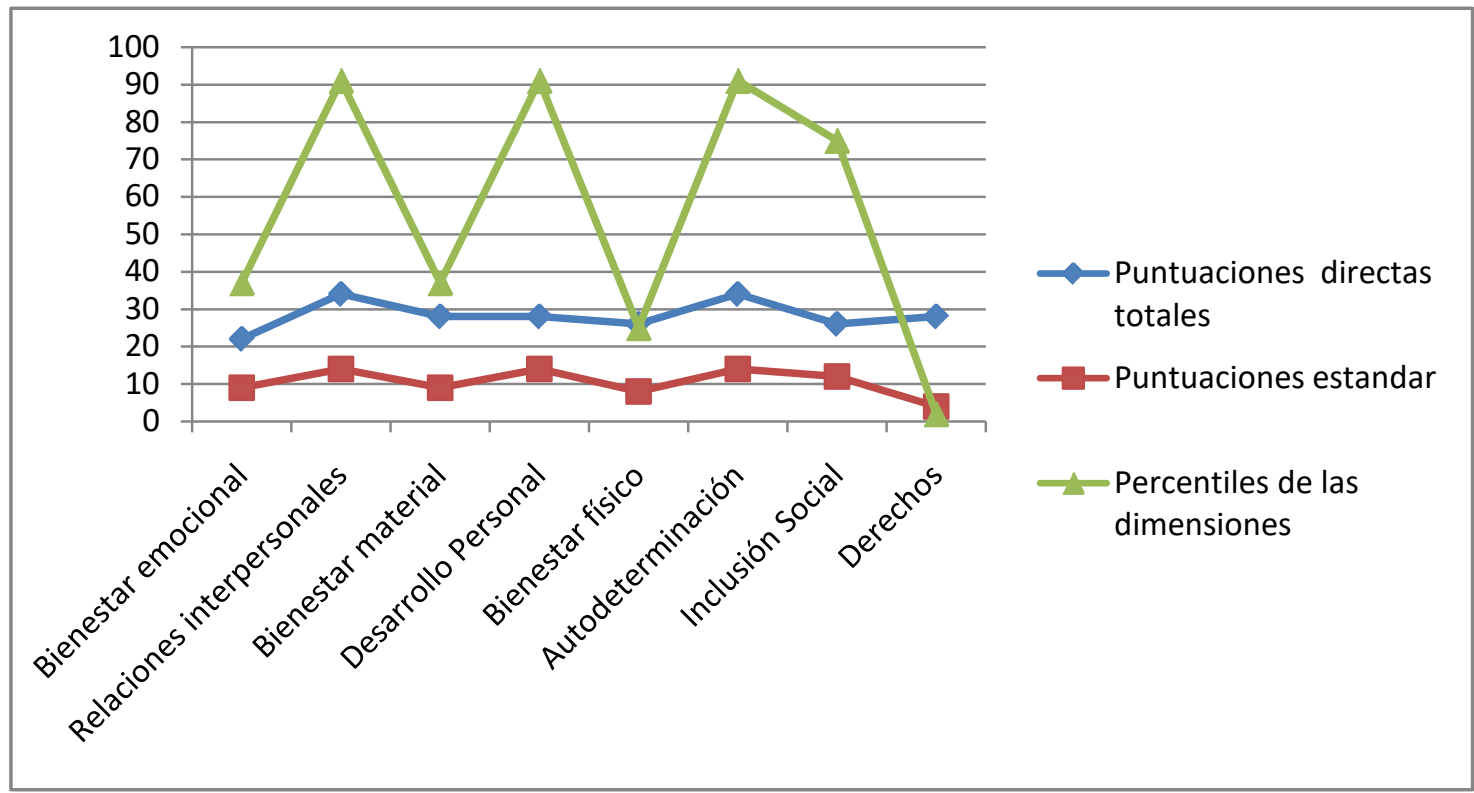

En el gráfico1 se observa el comportamiento de las diferentes dimensiones en el grupo de estudiantes. Las dimensiones de bienestar alcanzaron un percentil medio bajo; BE y BM se encuentran alineados en el mismo percentil (37), y no se observan diferencias significativas entre estas dos dimensiones y el BF, que alcanzó el percentil 25. Por otra parte, las dimensiones RI (91), DP (91), AU (91) e IS (75) puntuaron en percentiles altos. Resulta significativo que la dimensión DE se encuentra en el percentil más bajo registrado para este grupo, que alcanzó una puntuación de 2.

\section{Personal administrativo (PA)}

Tabla 3. Distribución de PA por sexo y grupo de edad

\begin{tabular}{|c|c|c|c|c|c|c|c|}
\hline \multicolumn{2}{|c|}{ SEXO } & \multicolumn{2}{|c|}{ GRUPO DE EDAD } & & & & TOTAL \\
\hline$F$ & M & 1 & 2 & 3 & 4 & 5 & \\
\hline & & De 18 a & De 26 a & De 36 a & De 46 a & Más de & \\
\hline & & 25 & 35 & 45 & 55 & 55 & \\
\hline 17 & 13 & 1 & 8 & 5 & 11 & 5 & 30 \\
\hline $57 \%$ & $43 \%$ & $3 \%$ & $26 \%$ & $17 \%$ & $37 \%$ & $17 \%$ & $100 \%$ \\
\hline
\end{tabular}


La muestra del personal administrativo estuvo compuesta por 30 empleados, 57\% (17 sujetos) del sexo femenino y 43\% (13 sujetos) del sexo masculino, distribuidos en los grupos de edad que van desde 18 a 25 hasta más de 55 años. El grupo de edad de 46 a 55 incluyó 11 sujetos (37\%); el de 26 a 35, 8 (26\%); en los grupos de 36 a 45 y más de 55, se reportaron 5 sujetos en cada uno de los grupos de edad que equivale al 17\% para cada uno, y para el grupo de 18 a 25 solo se reportó un sujeto (3\%).

Tabla 4. Puntuación Directa Total/Puntuación Estándar/Percentil de las Dimensiones/PA

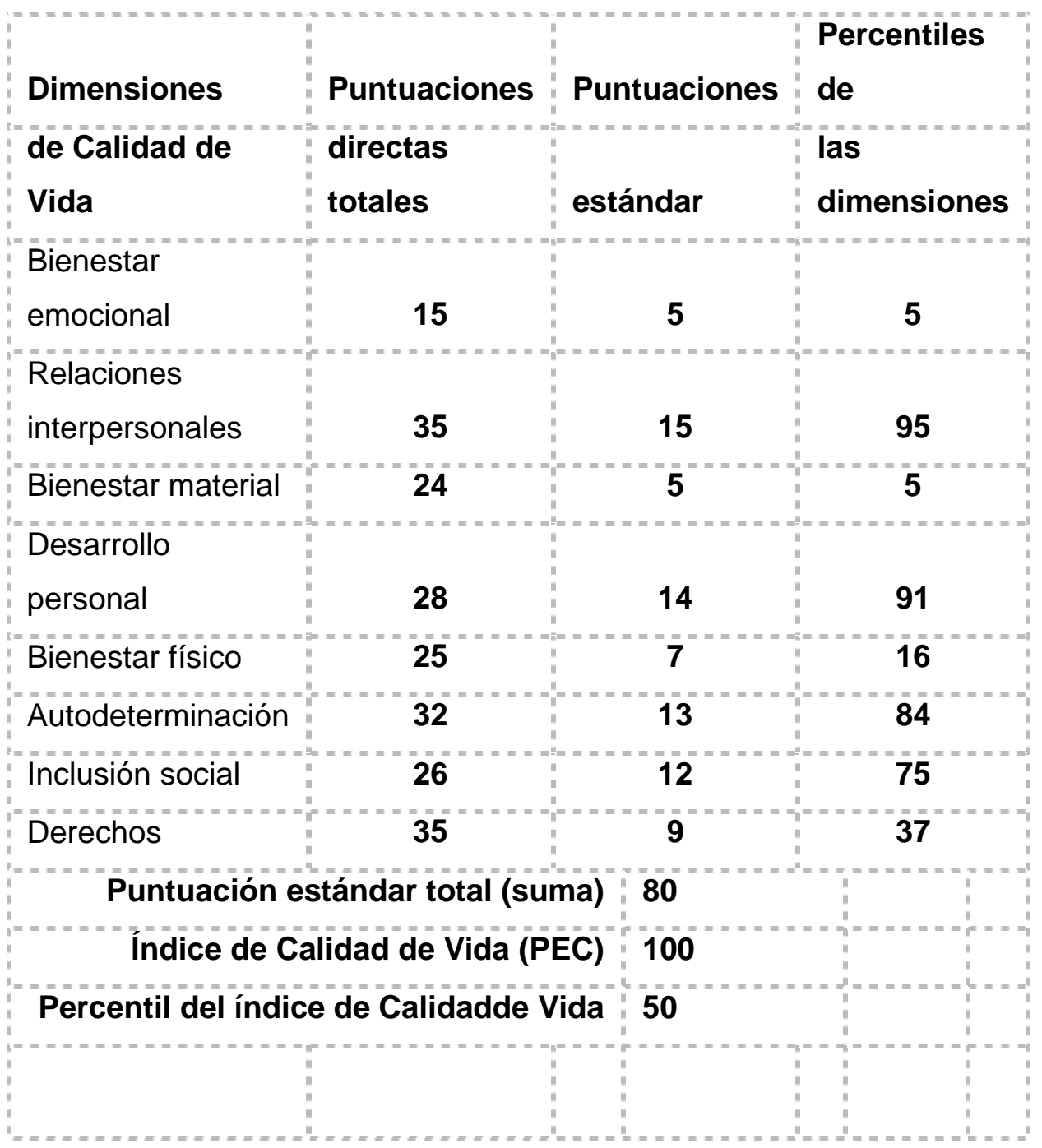

Como se observa en la tabla 4, el personal administrativo obtuvo una puntación estándar total de 80, y alcanzó un índice de Calidad de Vida de 100 y un percentil del índice de calidad de vida de 50 .

\section{Gráfico 2. Representación gráfica de la Puntuación Directa Total/Puntuación} Estándar/Percentil de las Dimensiones/PA 


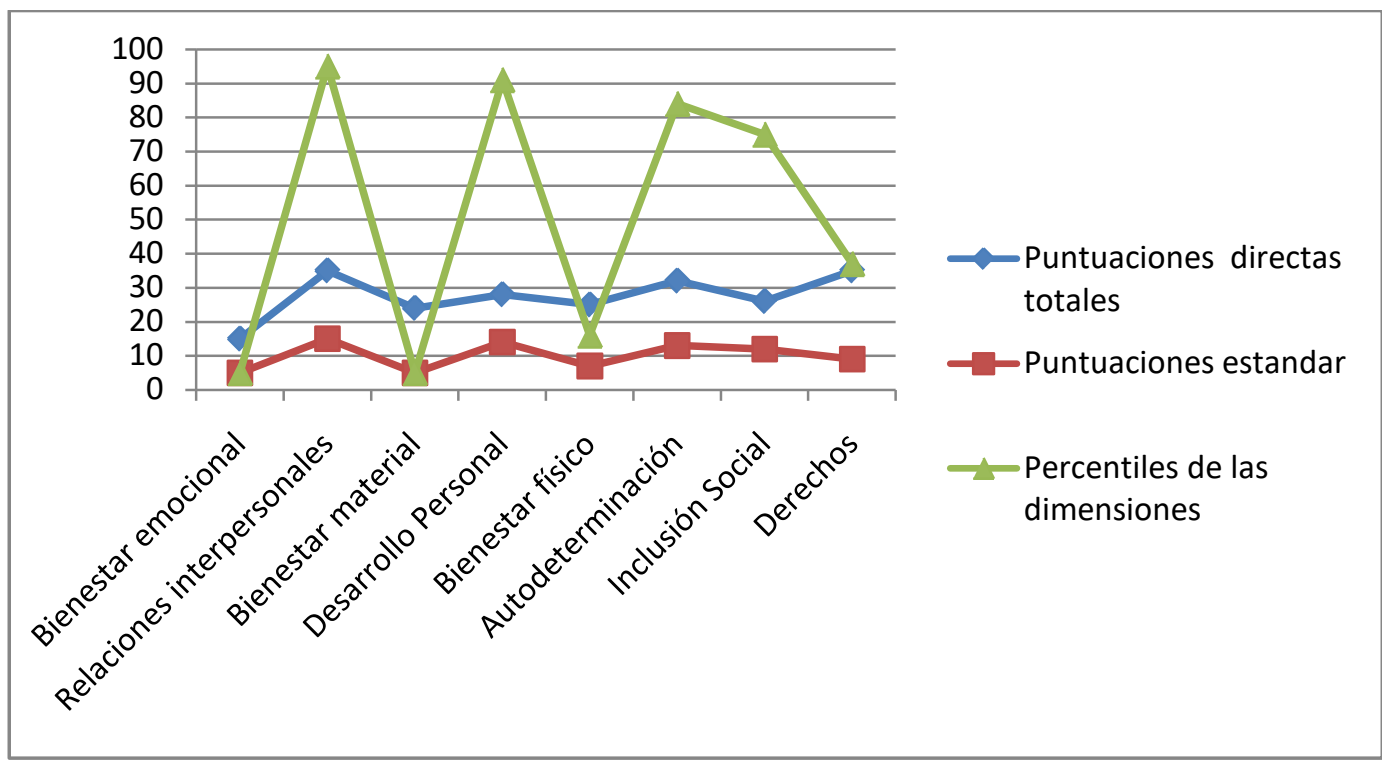

En el gráfico 2 observamos el comportamiento de las diferentes dimensiones en el grupo constituido por el personal administrativo. Las dimensiones de bienestar se puntuaron en percentiles bajos, el BE y el BM se alinearon en el mismo percentil (5) y el BF alcanzó el percentil 16. Las dimensiones RI (95), DP (91), AU (84) e IS (75) se puntuaron en percentiles altos. La dimensión DE se encuentra en un percentil medio bajo, dado que alcanzó una puntuación de 37.

\section{Profesor convencional (PC)}

Tabla 5. Distribución de PC por sexo y grupo de edad

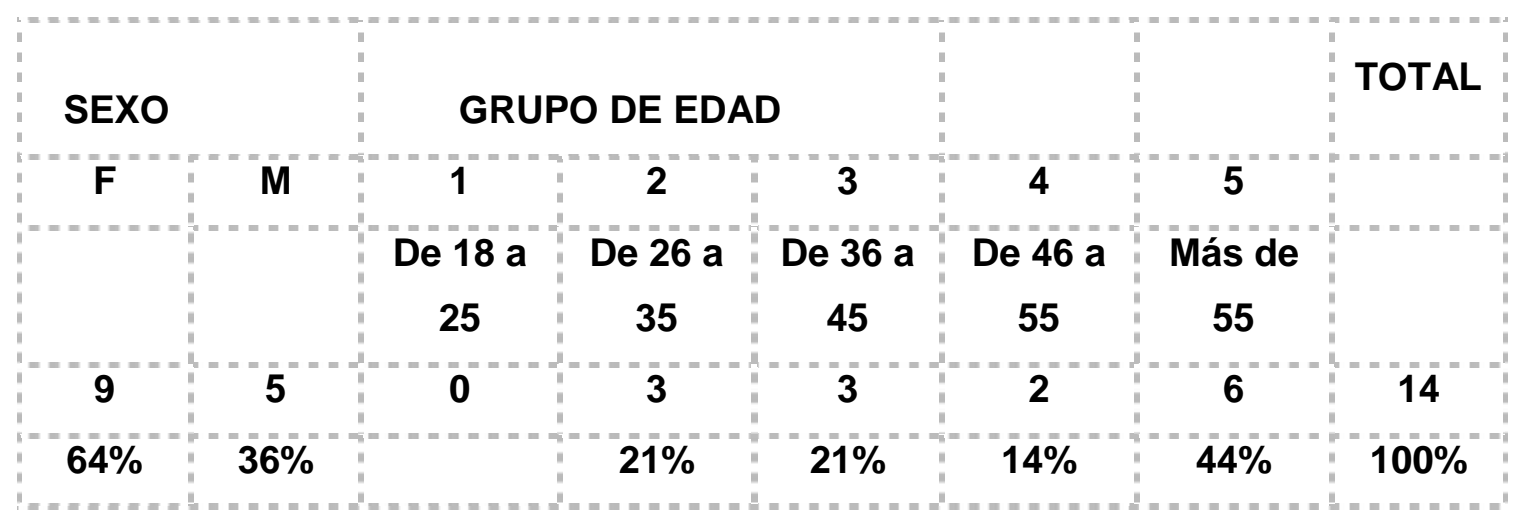

El total de la muestra de profesores convencionales fue de 14, de la queque 9 sujetos (64\%) correspondieron al sexo femenino y 5 (36\%) al sexo masculino. Se distribuyeron entre los grupos de edad de 26 a 35 y de más de 55 . El mayor número lo encontramos en el grupo de edad de más de 55, con 6 sujetos, lo que equivale al $44 \%$ del total de esta muestra; en los grupos de 26 a 35 y de 36 a 45, se reportaron 3 sujetos en cada uno de los grupos de edad, y esto correspondió al 21\%, y para el grupo de 46 a 55 se observa, en la tabla 7, que se reportaron 2 sujetos (14\%). 
Tabla 6. Puntuación Directa Total/Puntuación Estándar/Percentil de las Dimensiones/PC

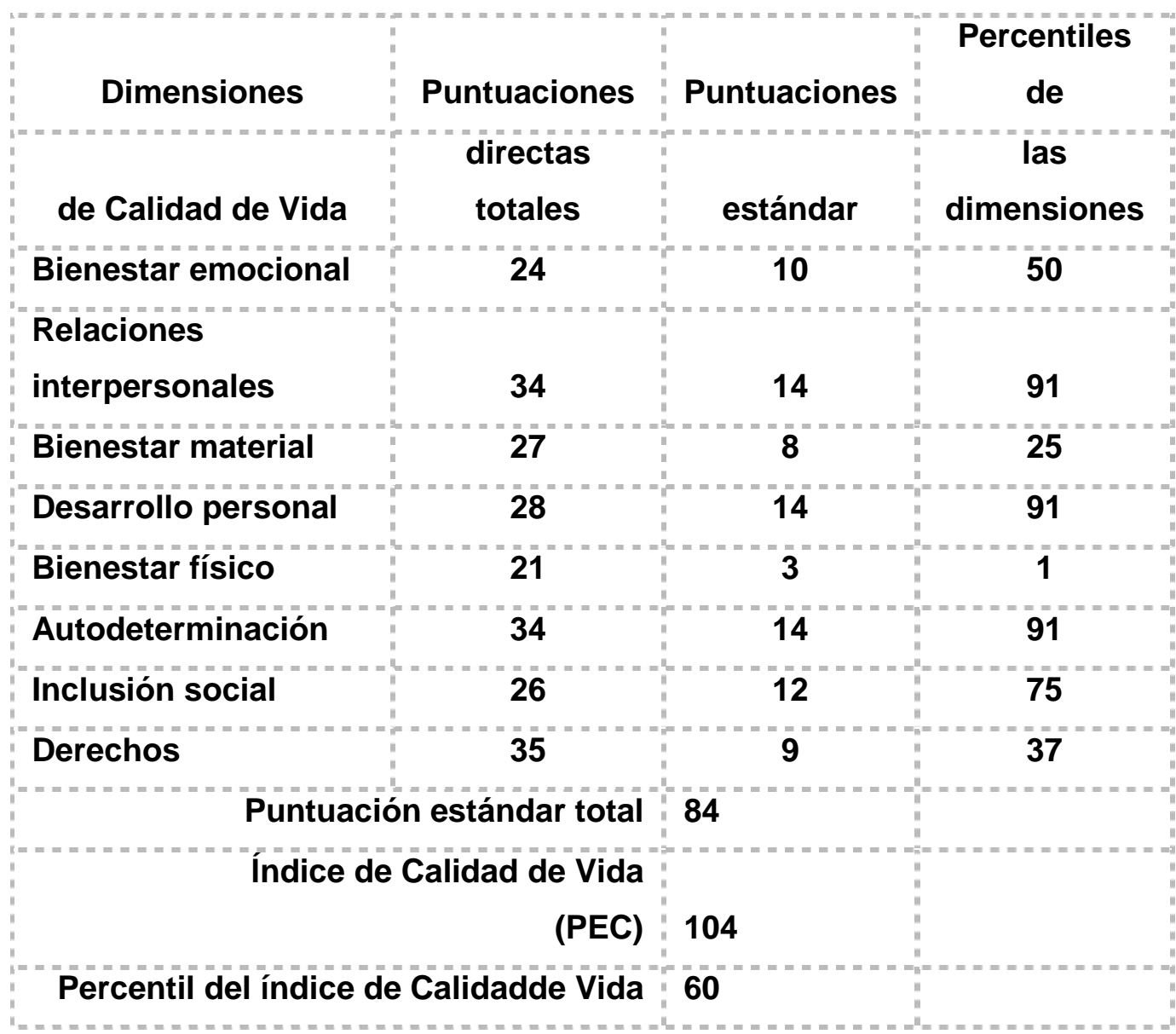

Observamos en la tabla 6 que los profesores convencionales alcanzaron una puntación estándar total de 84, un Índice de Calidad de Vidade 104 y un Percentil del índice de calidad de vida de 60 .

Gráfico 3. Representación gráfica de la Puntuación Directa Total/Puntuación Estándar/Percentil de las Dimensiones/PC 


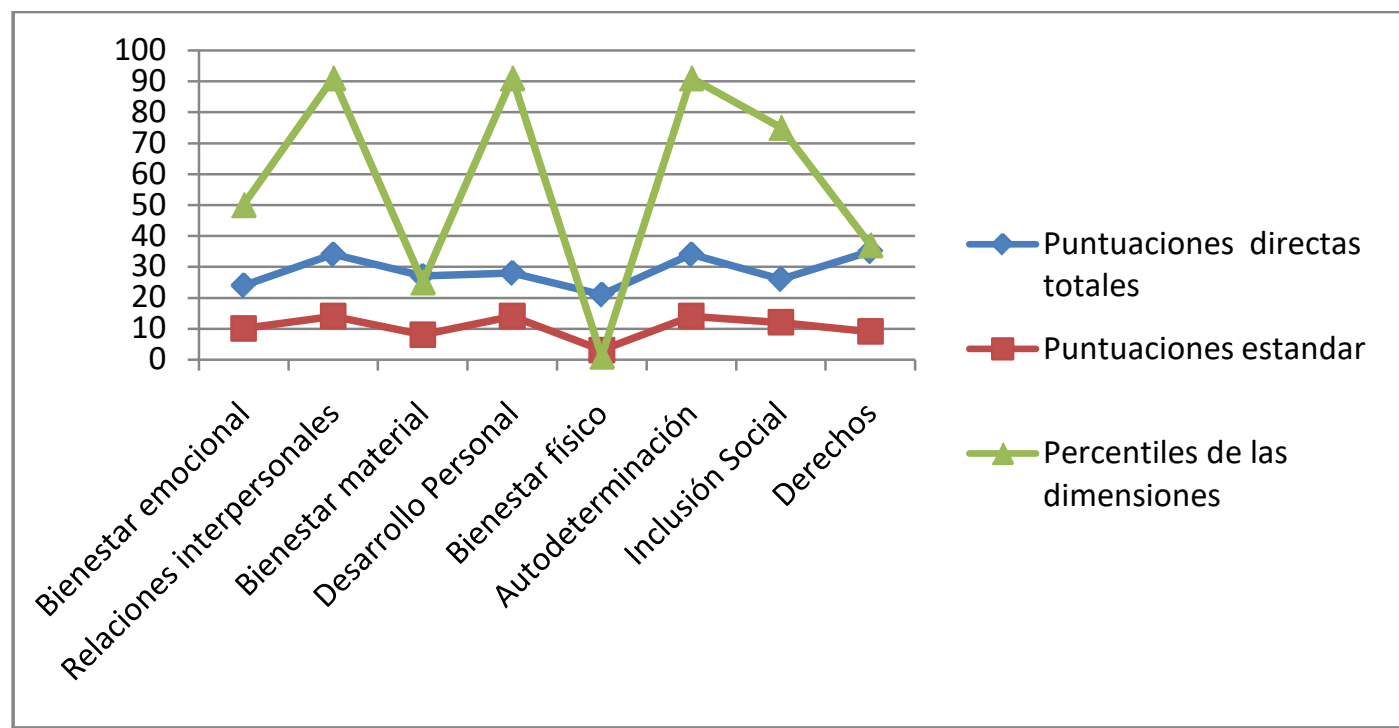

En el gráfico 3 podemos observar el comportamiento de las diferentes dimensiones en el grupo constituido por los profesores convencionales. El BE alcanzó el percentil 50, el BM se alinea en el percentil 25 y es significativo que en este grupo el BF se puntúa en el percentil 1. Las dimensiones RI (91), DP (91), AU (91) e IS (75) se puntuaron en percentiles altos. La dimensión DE se encuentra en un percentil medio bajo, dado que alcanzóuna puntuación de 37 .

\section{Profesor detiempo completo (PTC)}

Tabla 7. Distribución de PTC por sexo y grupo de edad

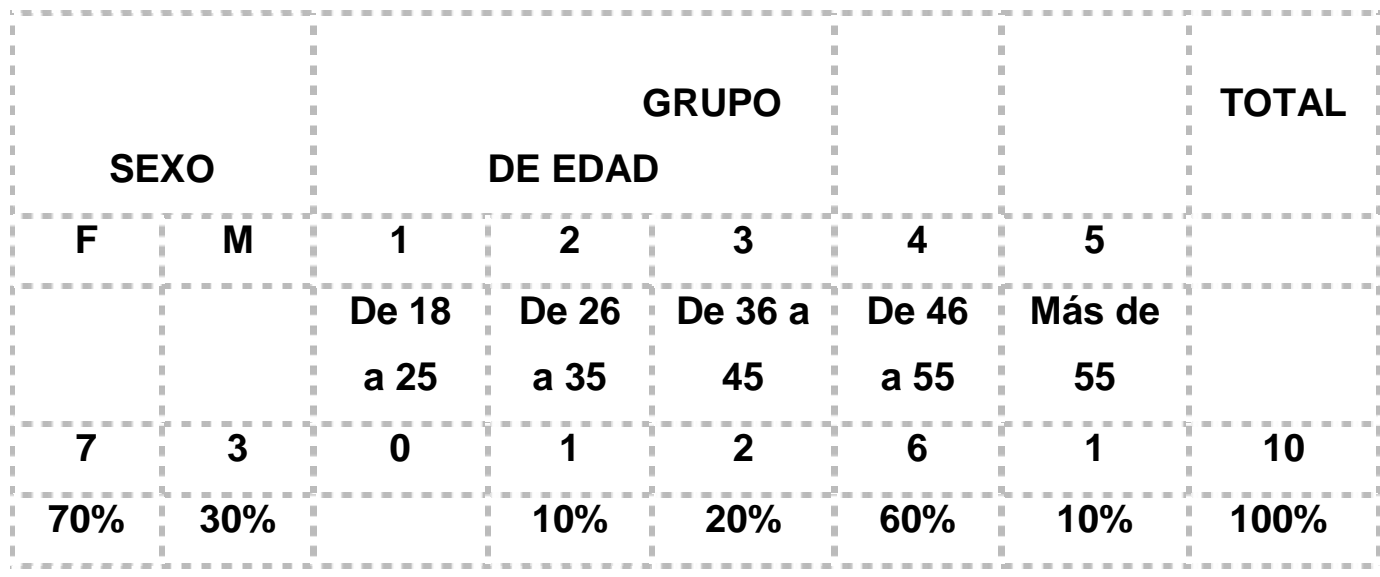

Para la muestra representativa de los profesores de tiempo completo se registraron 10 entrevistados, cuyas edades oscilaron entre los grupos de edad de 26 a 35 y más de 55. El mayor número de sujetos estuvo en el grupo de edad de 46 a 55, donde se encontraron 6 sujetos, lo que equivale al $60 \%$ del total de profesores de tiempo completo; en el grupo de 
36 a 45, se hallaron 2 sujetos (20\%) y en los grupos de 26 a 35 y de más de 55, se reportó unsujeto para cada uno de los grupos de edad, que corresponde al 10\% en ambos casos.

Tabla 8. Puntuación Directa Total/Puntuación Estándar/Percentil de las Dimensiones/PTC

\begin{tabular}{|c|c|c|c|}
\hline Dimensiones & Puntuaciones & Puntuaciones & Percentiles de \\
\hline de Calidad de Vida & directas totales & estándar & $\begin{array}{c}\text { las } \\
\text { dimensiones }\end{array}$ \\
\hline Bienestar emocional & 24 & 10 & 50 \\
\hline \multicolumn{4}{|l|}{ Relaciones } \\
\hline interpersonales & 34 & 14 & 91 \\
\hline Bienestar material & 27 & 8 & 25 \\
\hline Desarrollo personal & 30 & 15 & 95 \\
\hline Bienestar físico & 22 & 4 & 2 \\
\hline Autodeterminación & 32 & 13 & 84 \\
\hline Inclusión social & 26 & 12 & 75 \\
\hline Derechos & 35 & 9 & 37 \\
\hline \multicolumn{4}{|c|}{ Puntuación estándar total } \\
\hline \multicolumn{4}{|c|}{ Îndice de Calidad de } \\
\hline Percentil del índice & Calidadde Vida & 62 & \\
\hline
\end{tabular}

En la tabla 8, se observa que los profesores tiempo completo obtuvieron una puntación estándar total de 85, un índice de Calidad de Vida de 105 y un percentil del índice de Calidad de Vida de 62.

Gráfico 4. Representación gráfica de la Puntuación Directa Total/Puntuación Estándar/Percentil de las Dimensiones/PTC 


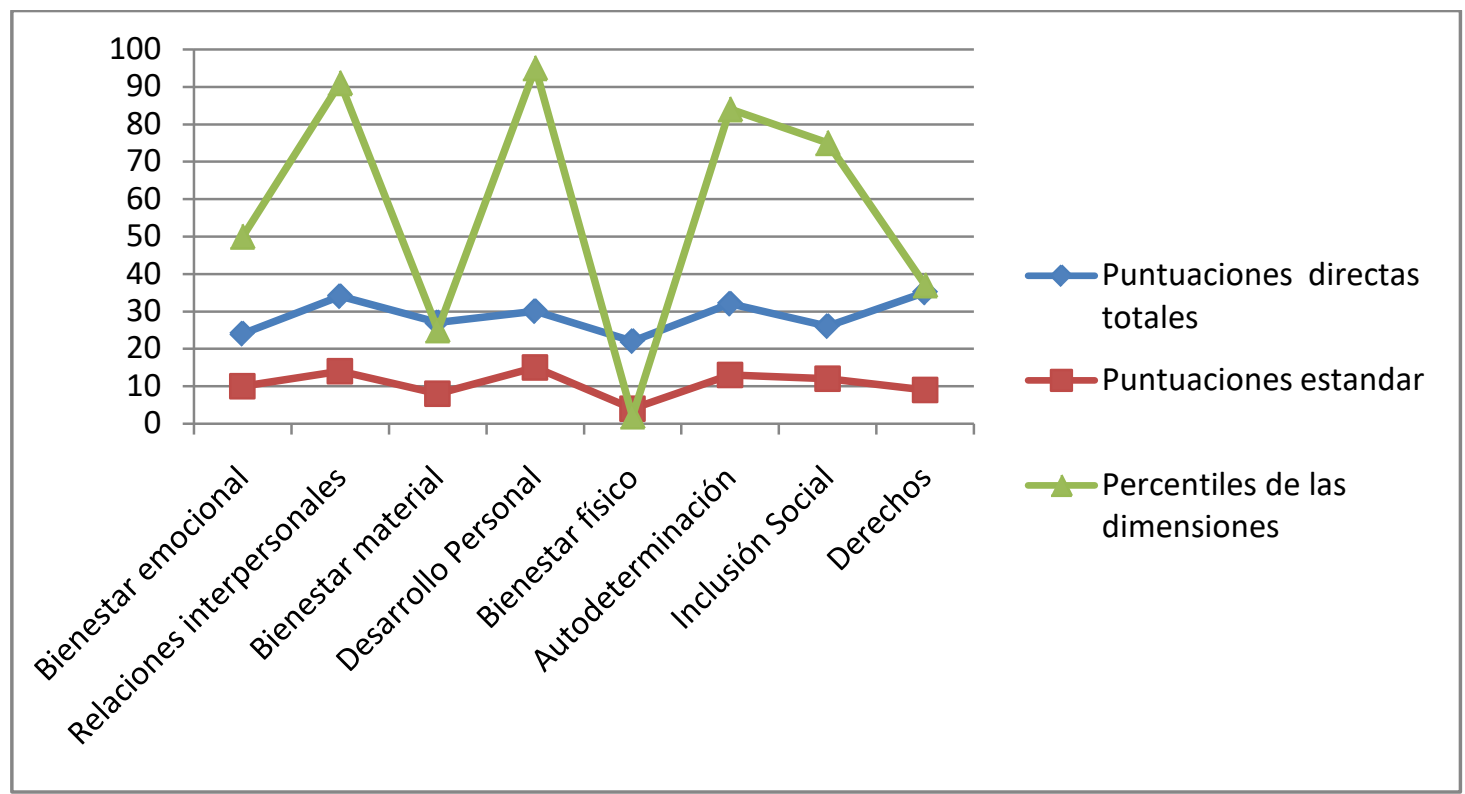

En el gráfico 4 se observa el comportamiento de las diferentes dimensiones en el grupo constituido por los profesores de tiempo completo. El BE alcanzó el percentil 50, el BM se alinea en el percentil 25 y el BF se puntúa, en este grupo, en el percentil 1. Las dimensiones RI (91), DP (95), AU (84) e IS (75) se puntuaron en percentiles altos. La dimensión DE se encuentra en un percentil medio bajo, con una puntuación de 37. Resalta la semejanza de puntuaciones en los percentiles de los profesores convencionales y los profesores de tiempo completo, a pesar de que no se observan diferencias significativas en ambos grupos.

Resultados de la medición de la Calidad de Vida que integran los datos recolectados en las diferentes muestras en el total de la población encuestada

Tabla 9. Distribución de la muestra total por sexo y grupo de edad

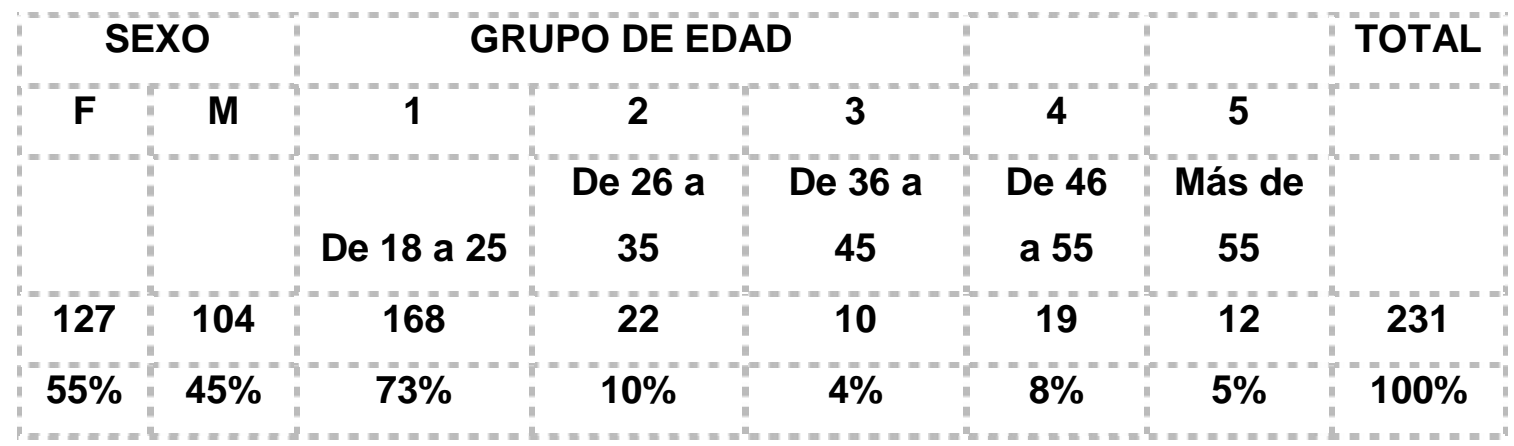

Se entrevistó a un total de 231 sujetos, con un porcentaje de mujeres ligeramente mayor al de varones - $55 \%$ del sexo femenino y $45 \%$ del masculino-. Los sujetos estuvieron distribuidos en grupos de edad de 18 a 25 y hasta más de 55 . En el grupo de18 a25 se reportaron 168 sujetos, que equivale al $73 \%$ del total; de 26 a 35 se observaron 22 
sujetos (10\%); de 36 a 45, 10 sujetos (4\%); de 46 a 55, un 8\% que equivale a 19 sujetos del total, y en el grupo de edad de más de 55 se reportaron 12 sujetos (5\%).

Tabla 10. Puntuación Directa Total/Puntuación Estándar/Percentil de Dimensiones/de muestra total

\begin{tabular}{|c|c|c|c|}
\hline Dimensiones & Puntuaciones & Puntuaciones & Percentiles de \\
\hline de Calidad de Vida & $\begin{array}{l}\text { directas } \\
\text { totales }\end{array}$ & estándar & $\begin{array}{l}\text { las } \\
\text { dimensiones }\end{array}$ \\
\hline Bienestar emocional & 25 & 11 & 63 \\
\hline $\begin{array}{l}\text { Relaciones } \\
\text { interpersonales }\end{array}$ & 33 & 14 & 91 \\
\hline Bienestar material & 27 & 8 & 25 \\
\hline Desarrollo personal & 29 & 15 & 95 \\
\hline Bienestar físico & 24 & 6 & 9 \\
\hline Autodeterminación & 33 & 14 & 91 \\
\hline Inclusión social & 26 & 12 & 75 \\
\hline Derechos & 33 & 8 & 25 \\
\hline \multicolumn{2}{|c|}{ Puntuación estándar total } & 88 & \\
\hline \multicolumn{2}{|c|}{ Índice de Calidad de Vida } & & \\
\hline & (PEC) & 107 & \\
\hline \multicolumn{2}{|c|}{ Percentil del índice de Calidad de } & & \\
\hline & Vida & 69 & \\
\hline
\end{tabular}

En la tabla 10 observamos que la muestra total alcanzó una puntación estándar total de 88, un índice de Calidad de Vida de 107 y un percentil del índice de Calidad de Vida de 69.

Gráfico 5. Representación gráfica de la Puntuación Directa Total/Puntuación Estándar/Percentil de las Dimensiones/de la muestra total 


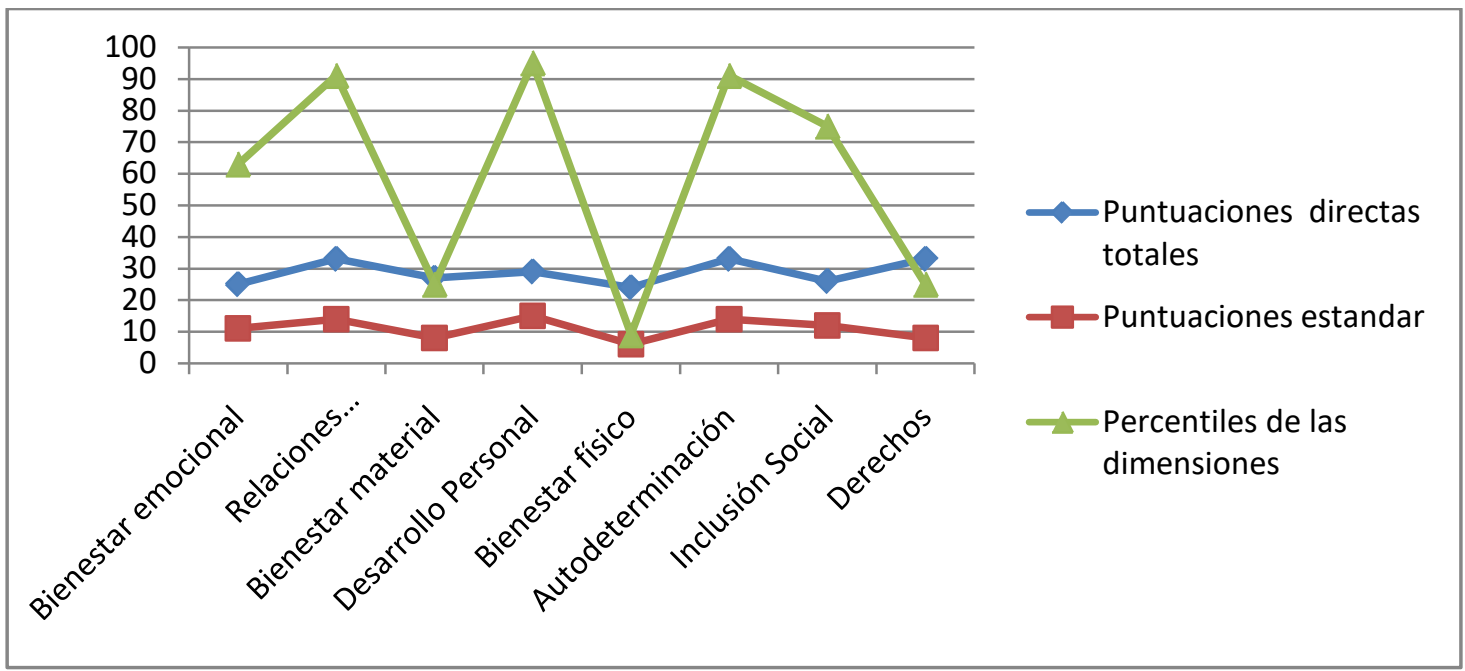

En el gráfico 5 puede observarse el comportamiento de las diferentes dimensiones en la muestra total. El BE alcanzó el percentil 63, el BM se puntuó más bajo, dado que se alineó en el percentil 25 y el BF se puntuó, para el grupo total, en el percentil 9. Las dimensiones RI (91), DP (95), AU (91) e IS (75) se puntuaron en percentiles altos. La dimensión DE se encontró en un percentil medio bajo, con una puntuación de 25 para el grupo total.

\section{Análisis de los resultados}

En cuanto al análisis de los datos, se siguió el método de análisis temático planteado por Glaser y Struss (1967), que implica la generación o descubrimiento de la teoría a partir de los datos recolectados. El análisis temático es el método fundamental de análisis cualitativo, sobre el que se basan otras formas cualitativas de análisis y que puede ser aplicado desde una amplia variedad de enfoques teóricos (Braun \& Clarke, 2006). Se analizaron los datos utilizando para su interpretación el baremo de la escala Gencat para la muestra general.

En primer lugar, puede afirmarse que los grupos no mostraron diferencias significativas en cuanto a las dimensiones RI, DP, AU e IS, ya que la puntuación de todos estaba en los percentiles altos. Esto nos permite inferir que los integrantes de la comunidad unimetana mantienen contactos sociales positivos y gratificantes con las personas que los rodean en los diferentes ámbitos donde se desenvuelven. Además, consideran que tienen acceso a las posibilidades de aprendizaje y a la oportunidad de adquirir conocimientos, así como acceso a las nuevas tecnologías. Se sienten integrados a la comunidad, apoyados e incluidos, con la oportunidad para participar en todos los lugares de uso común y en las actividades, sin distingo de rango laboral o social. Por otra parte, son capaces de decidir y elegir de manera autónoma aspectos referidos a su vida personal y laboral. En segundo lugar, las puntuaciones de las encuestas basadas en las respuestas de los participantes 
demuestran que poseen una calidad de vida media o baja en lo que respecta al bienestar emocional, psicológico, material y físico.

Respecto a la dimensión $\mathrm{BE}$, podemos inferir que los encuestados en general no se sienten tranquilos, se reportan como nerviosos y agobiados, y se sienten inseguros. En el caso del grupo de estudiantes y de profesores (convencionales y de tiempo completo), el bienestar emocional no ofrece diferencias significativas - los puntajes oscilan entre los percentiles 37 y 50 respectivamente- Sin embargo, en el caso del personal administrativo, es alarmante el bajo percentil en esta dimensión, lo que indica que su bienestar emocional está muy debilitado.

Para la dimensión de bienestar material (BM), al igual que en el BE, los grupos de estudiantes y profesores (convencionales y de tiempo completo) se ubicaron en percentiles medios bajos, lo que indica que no disponen del dinero suficiente para solventar sus necesidades y gustos, sumado a que no disponen de un empleo con una remuneración que les permita ahorrar y/o adquirir bienes materiales. Nuevamente, resulta significativo que en esta dimensión el grupo del personal administrativo se ubicó en el percentil 5, igual que en la dimensión BE. Esto indica que sus ingresos no cubren sus necesidades básicas y tienen menores oportunidades de adquisición de bienes materiales y de capacidad de ahorro en comparación a todos los integrantes de la muestra.

En cuanto a la dimensión BF, se observaron diferencias significativas entre los grupos. Los estudiantes puntuaron en el percentil 25 y el personal administrativo en el percentil 16; ambos grupos se encuentran en percentiles medios-bajos, lo que indica que no se sienten con buena salud y que no están practicando hábitos de alimentación saludable, con alteraciones del sueño y un inadecuado estado de salud. Por otra parte, el grupo de profesores, tanto convencionales como de tiempo completo, puntuaron para esta dimensión en el percentil 1 .Este puntaje es indicio de que para ambos grupos de profesores el bienestar físico se encuentra en situación crítica, y que su salud y su alimentación se han visto muy comprometidas para el momento en que participaron en la encuesta. Esta es la dimensión más comprometida de toda la muestra, donde se reportaron niveles bajos y muy bajos de puntuación percentil.

La dimensión DE se ubicó, para los grupos de personal administrativo y profesores (convencionales y de tiempo completo) en un percentil medio-bajo, con una puntuación de 37. Debe considerarse esta puntuación como indicador de atención, ya que reporta que esta población se considera vulnerada en el ejercicio de sus derechos y está exigiendo que sea considerada igual que el resto de la gente, que se la trate del mismo modo, que se respete su forma de ser, sus opiniones y deseos. El caso de los estudiantes es alarmante, ya que consideran que sus derechos han sido vulnerados hasta el punto de alcanzar un 
percentil de 2 en esta dimensión. Es la dimensión más baja registrada para este grupo e indica que se perciben irrespetados, no escuchados, no tomados en cuenta.

El comportamiento de las dimensiones RI (91), DP (95), AU (91) e IS (75) en la muestra total reflejaron percentiles altos, lo que coincide con las puntuaciones de los grupos considerados individualmente. La dimensión de bienestar emocional alcanzó el percentil 63 (medio-alto) y el bienestar material y la dimensión DE quedaron representadas en un percentil medio-bajo (25), por último, la dimensión BF puntuó, para el grupo total, en el percentil 9.

En relación con el percentil superior o inferior obtenido, se evidencia que existen tanto niveles bajos como niveles altos de calidad de vida en el contexto donde se desenvuelve la población estudiada. Algunos de los puntajes más bajos pueden representar a las personas que se encuentran en las condiciones más precarias, que carecende condiciones ambientales más apropiadas y de los recursos necesarios para obtener un adecuado bienestar. En relación a los puntajes más altos, pueden hacer referencia a los sujetos que cuentan con mejores condiciones, lo cual puede motivarlos a percibir de manera positiva el contexto en que se desenvuelven a pesar de que en realidad las condiciones del mismo no sean las más adecuadas.

\section{Conclusiones}

Los resultados indicaron que las puntuaciones obtenidas oscilaron de bajas a altas, dado que en cuatro de los casos superaron el punto medio teórico de las escalas, con percentiles altos, y en donde las dimensiones se obtuvieron puntajes medios, tales como en Bienestar Emocional y Material. Los peores resultados se observaron en las dimensiones Bienestar Físico y Derechos.

Si tuviéramos que calificar a las personas evaluadas en los cuatro grupos en función de su puntuación total en la escala (calidad de vida alta, media y baja), el porcentaje se engloba en la categoría de calidad de vida media con tendencia a baja. Se debe tener en cuenta que de las ocho dimensiones, cuatro han reflejado percentiles altos en los cuatro grupos y las cuatro restantes se han comportado de manera variable en los diferentes grupos, entre percentiles medio bajos y muy bajos.

Las escalas que más peso muestran a la hora de discriminar entre personas con calidad de vida alta, media y baja son las dimensiones de Bienestar Emocional, Material, Físico y Derechos, seguida con cierta distancia por las dimensiones de Relaciones Interpersonales, Desarrollo Personal, Autodeterminación e Inclusión Social, las dimensiones menos consideradas para el caso estudiado.

La vulnerabilidad reflejada en algunas de las dimensiones medidas en la muestra de este estudio sobrepasa los límites de lo razonable por el tipo de población encuestada. La 
muestra se encuentra en un país con una situación desbordada, que ha generado cambios importantes y desmedidos en el desarrollo de sus procesos diarios de vida, lo que dio lugar a inestabilidad económica y emocional, deterioro de salud física, disminución de adquisición de materiales, vulneración de los derechos, inseguridad, entre otras consecuencias.

Los puntajes reflejados por la población de estudio en cuanto a las dimensiones de bienestar son signo de que deben mejorarse las circunstancias en que se desenvuelven los sujetos a fin de que se generen las condiciones que favorezcan el alcance de un mayor bienestar emocional y físico. Respecto de las mismas dimensiones, en el caso del grupo del personal administrativo, se deberán establecer a la mayor brevedad posible y con alta precisión las necesidades reales y sentidas del grupo, a fin de diseñar y desarrollar programas que hagan superarlos bajos percentiles arrojados en la aplicación de la escala.

Se hace evidente la pertinencia de la utilización de la escala siempre y cuando sea ajustada a las características de la población de estudio. Al revisar investigaciones en diferentes tipos de muestras, concluimos que la escala es válida y fiable, como lo reportan Arias, et al. (2010). Sin embargo, podemos decir que para cada población es aconsejable revisarla y generar un modelo que se adapte a las características propias de la muestra que se investiga, como lo proponen Henao, et al. (2015), para quienes fue necesario generar una versión de la escala adaptada a la población colombiana, para el caso de la discapacidad intelectual. Por otra parte, reportan que la escala presenta adecuados niveles de validez y fiabilidad, lo que refuerza la conclusión a laque llegaron.

También se hace necesario trabajar con las muestras por estratos y en diferentes momentos a lo largo de diferentes períodos de tiempo, como lo consideran los autores Campos-Vásquez y Lara (2012).

En todos los casos de investigación revisados, la mayoría de los ítems de la escala se ajustan al modelo para aplicar en cada muestra, a pesar de ser muestras totalmente diferentes.

La limitación más importante es reportada por Benito, et al. (2016), que informan que el tamaño de la muestra es directamente proporcional a la confiabilidad de la escala, de allí que cuanto menor es el tamaño de la muestra, la confiabilidad disminuye de igual forma. $\mathrm{A}$ pesar de todo se puede concluir que la escala posee evidencias suficientes de fiabilidad como para evaluar la calidad de vida de las personas.

\section{Propuestas basadas en la superación de las condiciones en las dimensiones que la muestra reportó como de menor satisfacción}

En el caso del grupo conformado por los estudiantes, la dimensión corresponde a los derechos: i) Realizar grupos focales para conocer en mayor profundidad las razones por las cuales se perciben tan vulnerables frente al respeto de sus derechos e identificar los 
ámbitos y las áreas críticas, ii) Llevar a cabo talleres formativos sobre la defensa de los derechos humanos, iii) Diseñar campañas internas (decanato de estudiantes, agrupaciones estudiantiles, consejos de estudiantes, federación de estudiantes) sobre el ejercicio de sus derechos, iv) Elaborar una campaña informativa sobre los reglamentos estudiantiles.

Para el caso del grupo constituido por el personal administrativo y los profesores, las dimensiones reportadas como de menor satisfacción fueron: bienestar emocional, bienestar material y bienestar físico: i) Hacer un relevamiento de necesidades discriminado por grupo a través de "Encuesta Fácil". ii) Realizar talleres de desarrollo personal que brinden herramientas para afrontar las diversas situaciones que reportan estar atravesando. iii) Elevar sugerencias a la Dirección de Capital Humano sobre la realización de un sondeo del clima laboral, jornadas preventivas de salud, revisión de la alta siniestralidad del HCM (Hospitalización, Cirugía y Maternidad) que ha estado reportando con alta frecuencia el personal. iv) Revisarperiódicamente de las acciones compensatorias en el ámbito económico que la institución ha estado otorgando al personal.

\section{Recomendaciones y limitaciones}

Diseñar y desarrollar proyectos que mejoren la percepción y las condiciones sociales en las que se encuentran los sujetos actualmente, y establecer mecanismos de seguimiento que velen por mantener óptimas condiciones en el mejoramiento de la calidad de vida.

Se recomienda la validación del modelo de las ocho dimensiones en la población venezolana.

Para futuros estudios, es recomendable investigar una muestra más representativa y para ello se recomienda la ampliación del estudio con muestras similares.

Aplicar la encuesta nuevamente y comparar resultados de acuerdo con la actual situación del país, ya que se debe tener en cuenta que la evaluación de la calidad de vida puede variar no solo en cuanto a las puntuaciones obtenidas en las distintas dimensiones, sino que también sufre variaciones en el transcurso del tiempo y en función de los distintos acontecimientos vitales en la vida de las personas. De allí que se recomienda evaluar periódicamente a cada persona con el objetivo de obtener información actualizada sobre su calidad de vida y especialmente si se ha implementado un plan de intervención cuyo objetivo básico sea el de mejorar alguna de las dimensiones.

Entre las limitaciones podemos resaltar el reducido tamaño de la muestra, más concretamente, el reducido número de personas que componen cada grupo en el análisis por variables, que limita el análisis de diferencias mediante contrastes estadísticos. Tal análisis constituye una línea futura de investigación a abordar.

Se prevé administrar nuevamente la escala para valorar el impacto de los programas que se desarrollen y los cambios que se implementen a partir de las sugerencias del 
presente trabajo, y constatar si se producen mejoras en la calidad de vida de la población estudio.

\section{Referencias bibliográficas}

Andrews, F. M. \&Withey, S. B. (1976). Social Indicators of well-being. Americans' Perceptions of Life Quality. Nueva York: Plenum Press.

Arostegui, I. (1998). Evaluación de la calidad de vida en personas adultas con retraso mental en la comunidad autónoma del País Vasco. Bilbao: Universidad de Deusto.

Benito, E., Mora, C. y Macía, A. (2016). Propiedades psicométricas de la Escala Gencat de calidad de vida en una muestra de personas sin hogar. Siglo Cero: Revistas sobre discapacidad intelectual, 47(1), 23-36.

Blanco, A. (1985). La calidad de vida, supuestos psicosociales. En J.F. Morales y cols. Psicología Social Aplicada, DDB.

Bramston, P. (2002). "Subjective Quality of Life: The Affective Dimension",enGullone, E.\& Cummins, R. (eds) Social Indicators Research Vol. 16. Kluwer Academic Publishers. PaísesBajos, 47-62.

Braun, V. \& Clarke, V. (2006). "Using Thematic Analysis in Psychology". Qualitative Research in Psychology, 3(2), 77-101.

Calman, K. C. (1987). "Definitions and dimensions of Quality of Life". En Aaronson, N. K.; Beckmann, I.M. (Eds.). The Quality of Life in Cancer Patients. Nueva York: Raven Press, 1-10.

Campbell, A. (1976). "Subjective meausures of Well-being". American Psychologist, 31(2), 117-24.

Campbell, A.; Converse, P., \& Rodgers, W. (1976). The Quality of American Life: Perceptions, Evaluations and Satisfactions. Nueva York: Rusell Sage Foundation.

Campos-Vázquez, R.M. \& Lara J. (2012). "Self-selection Patterns among Return Migrants: México 1990-2010". IZA Journal of Development and Migration 1:8.

Casas, F. (1999). "Calidad de vida y calidad humana". Revista Papeles del Psicólogo, Nº 74. Madrid, Colegio de Psicólogos de Madrid, pp. 46-54.

Contreras, M.H. y Cordero, V.A. (1994). Ambiente, desarrollo sostenible y calidad de vida. Ediciones Miguel Ángel García e Hijo. Caracas, Venezuela.

Cummins, R. y Cahill J. (2000). "Avances en la comprensión de la calidad de vida subjetiva". En Intervención psicosocial. Revista sobre igualdad y calidad de vida. Vol. 9(2) Colegio Oficial de Psicólogos de Madrid. 185-198.

Cummins, R. A. (2005). "Moving from the Quality of Life Concept to a Theory". Journal ofIntellectualDisabilityResearch, 49 (10):699-706. 
Encuesta Condiciones de Vida de la Población Venezolana (ENCOVI) (2016). Universidad Católica Andrés Bello, Universidad Central de Venezuela y Universidad Simón Bolívar, Caracas, Venezuela.

Ferris, A. (2006). "A Theory of Social Structure and the Quality of Life". EnApplied Research in Quality of Life Vol. 1. Springer. PaísesBajos, 117-123.

García-Riaño, D. (1991). "Calidad de vida. Aproximación histórico-conceptual". Boletín de Psicología, 30:55-94.

Glaser, B. y Struss, A. (1967). El desarrollo de la teoría fundada. Chicago: Aldine.

Gullone, E \& Cummins, R. (2002). (eds) Social Indicators Research Vol. 16. Países Bajos: Kluwer Academic Publishers.

Haas, B. (1999). "Clarification and Integration of Similar Quality of Life Concepts". Journal of Nursing Scholarships, 31(3), 215 - 220.

Henao, C.P., Verdugo, M.A. y Córdoba, L. (2015). "Adaptación para Colombia de la Escala INICO-FEAPS de evaluación de calidad de vida de personas con discapacidad intelectual". Revista Facultad de Medicina, 63 (4), 677-86

Jaimes, E. y Carballo, N. (2014). Modelo modificado de calidad de vida, UNELLEZ VIPI, San Carlos, Cojedes. Mimeografiado no editado.

Kreitler, S. \&Nive, D. (2007). "Quality of Life and Coping in Chronic Pain Patients". en: Kreitler, S, Beltuti, D., Lamberto, A. yNiv, D. (eds.), The Handbook of Chronic Pain. Pp. 77-99. USA: Nova.

Lawton, M.P. (1975). "The Philadelphia Geriatric Center Morale Scale: A Revision". JournalofGerontology, 30, 85-89

Levi, L. y Anderson, L. (1980). La tensión psicosocial. Población, ambiente y calidad de vida. México: El Manual Moderno.

Nava, M. G. (2012). "La calidad de vida: análisis multidimensional" Enfermería Neurológica, México, 11(3), 129-137.

OMS (1996). Foro Mundial de la Salud. Ginebra, Organización Mundial de la Salud (OMS).

Osorio, P. P., Torrejón, M. J. y M. S. Anigstein (2011). "Calidad de vida en personas mayores en Chile" Revista Mad-Universidad de Chile. 24, 61-77.

Palomba, R. (2002). Calidad de vida: conceptos y medidas. Santiago de Chile, Celade/División de Población, CEPAL.

Provea (2015). Informe Anual Enero-Diciembre. Programa Venezolano de EducaciónAcción en Derechos Humanos (PROVEA).

Raymundo, M. y Jaimes, E. (2015). Modelo para valorar la calidad de vida de los egresados del programa nacional de aprendizaje del INCES (Instituto Nacional de Capacitación Educativa Socialista), Estado Cojedes, Venezuela. 
Rojas, M. y Martínez, I. (2012). Medición, investigación e incorporación a la política pública del bienestar subjetivo: América Latina. Foro Consultivo Científico y Tecnológico (FCCyT). Reporte de la Comisión para el Estudio y la Promoción del Bienestar en América Latina, México.

Schalock, R.L. (1997). Quality of life. Vol. II: Application to persons with disabilities. Washington, D.C.: American Association on Mental Retardation. Washington: AAMR.

Schalock, R. L. (2005). Introduction and Overview to the Special Issue. Journal of Intellectual Disability Research, 49 (9), 695-698.

Schalock, R. L. y Verdugo, M. Á. (2003). Calidad de vida. Manual para profesionales de la educación, salud y servicios sociales. Madrid, España: Alianza Editorial.

Schalock, R. L. y Verdugo, M. A. (2007). "El concepto de calidad de vida en los servicios y apoyos para personas con discapacidad intelectual". Siglo Cero, Revista Española sobre Discapacidad Intelectual 38 (4), 21-36.

Shin, D.C. y Johnson, D.M. (1978). "Avowed Happiness as an Overall Assessment of the Quality of Life".Social IndicatorsResearch, 5, 475-492.

Tonon, G. (2003). Calidad de vida y desgaste profesional: una mirada del síndrome de burnout. Buenos Aires, Argentina: Espacio Editorial, pp 93.

Tonon, G. (2009) "Los estudios sobre calidad de vida y la perspectiva de la psicología positiva" Revista Psicodebate. 10, 73-81.

Topolski T, Edwards T, Patrick D. (2002). User's Manual and Interpretacion guide for the Youth Quality of Life (YQOL) Instruments. Seattle, WA: University of Washington, Department of Health Services.

Urzúa A, Caqueo-Urízar A. (2012). "Calidad de vida: una revisión teórica del concepto". Terapia Psicológica, Vol.30 (1), 61-71.

Verdugo, M. Á., Arias, B., Gómez, L. E. y Schalock, R. L. (2009). Escala GENCAT. Manual de aplicación de la Escala GENCAT de Calidad de Vida. Barcelona:Departamento de Acción Social y Ciudadanía de la Generalitat de Catalunya.

[1] Psicólogo egresado de la UCAB, magíster S/T análisis experimental de la conducta en la UCV. Músicoterapeuta egresado de la UC, especialista en Tecnología, Aprendizaje y Conocimiento en la UNIMET y coach de bienestar. Investigador en proyectos de investigación-acción-participación en CENDIF-UNIMET (2016-2017). Profesor detiempo completo en UNIMET desde 1997. Jefe de departamento de Desarrollo Integral (hasta 2016). Coautor de publicaciones, ponencias.

[2] Socióloga por la UCV (1996). Investigador en proyectos de investigación-acciónparticipación en CENDIF-UNIMET, (1997-2017). Docente de tiempo completo en 
laUNIMET. Jefe del departamento de Desarrollo integral (2016-2017). Arbitraje de revistas y libros en el área de especialización. Coautora de publicaciones, ponencias, manuales de capacitación. Recibió premios y reconocimientos a nivel nacional e internacional.

[3] Psicóloga Social por la UCV (1979). Directora del Centro de Investigaciones CENDIFUNIMET.Ha trabajado en el diseño, la ejecución y la evaluación de proyectos de investigación-acción-participación (1986-2017). Docente de tiempo completo en la UNIMET. Arbitraje de revistas y libros en el área de especialización. Autora y coautora de publicaciones, ponencias, manuales de capacitación. Ha recibido premios y reconocimientos a nivel nacional e internacional.

[4] Se aclara que el personal académico de la Universidad Metropolitana se caracteriza según la dedicación, la vinculación contractual con la universidad y las funciones universitarias. El artículo 6 delReglamento de caracterización, evaluación y compensación económica del personal académico dice:

"Según su dedicación[...] 1. El personal académico a tiempo completo desarrolla funciones universitarias durante una jornada laboral de treinta y seis horas distribuidas a lo largo de la semana, en función de las necesidades institucionales. 2. El personal académico a tiempo parcial desarrolla una parte de las funciones universitarias, según horas de dedicación acordadas".

Por ello, los profesores se presentaron en dosgrupos diferentes. Además de ser distintospara la institución, se consideró muy importante diferenciarlos para tomar decisiones adecuadas a cada grupo en específico. 